\title{
Visualización de datos de movilidad en la Ciudad de México, Santa Fe \\ Sleyter Angulo-Chavarría ${ }^{1}$
}

Angulo-Chavarría, S. Visualización de datos de movilidad en la ciudad de México, Santa Fe. Tecnología en Marcha. Vol. 33, especial Movilidad estudiantil. Pág 203-214.

doi) https://doi.org/10.18845/tm.v33i7.5494 


\title{
Palabras clave
}

Ciudades inteligentes; rápida urbanización; incremento acelerado de la población, congestionamiento vial; datos abiertos; patrones de comportamiento; sistema multi-pantalla.

\section{Resumen}

Las ciudades inteligentes emergen como una estrategia para poder combatir la rápida urbanización, el incremento acelerado de la población y el congestionamiento vial. Este articulo propone una solución basada en la recolección de datos abiertos, que nos permita encontrar variables o patrones de comportamiento, para luego ser analizados en un sistema multi-pantalla. A través de este sistema, se pueden generar distintos escenarios con datos reales como: el clima, eventos en la ciudad, fechas de pago; permitiendo a los tomadores de decisiones realizar analisis criticos de la situación expuesta.

\section{Keywords}

Smart cities; accelerated urbanization; rapid population growth; traffic jam; open data; behavior patterns; multi-screen system; decision makers.

\begin{abstract}
The smart cities are emerging as a strategy to mitigate the problems generated by accelerated urbanization, rapid population growth and traffic jam. This paper proposes a solution based on the collection of open data, which allows us to find variables or behaviour patterns, and then be analyzed in a multi-screen system. Using this tool, different scenarios can be generated with real data such as: weather, city events, payment days; allowing to decision makers to perform critical analyzes of the situation described.
\end{abstract}

\section{Introducción}

Actualmente, más de la mitad de la población mundial vive en áreas urbanas [5-7]. La migración de personas de áreas rurales a urbanas se prevé que siga en aumento en los próximos años. Ante está situación se empiezan a crear una serie de problemas como dificultad de la gestión de residuos, escases de recursos, contaminación, enfermedades, congestionamiento vial e infraestructuras deterioradas [8]. Otro tipo de problemas que existen son los relacionados con diferentes interesados, altos niveles de interdependencia, complejidad social y política. Convirtiendo los problemas de la ciudad en terribles y enredados $[4,9,15,17]$. Ante este panorama surge un problema de cómo las ciudades, las regiones circundantes y las áreas rurales pueden evolucionar hacia ecosistemas de innovación sostenibles, abiertos e impulsados por el usuario, para promover la investigación y experimentaciones futuras de servicios impulsados por el usuario y cómo pueden acelerar el ciclo de investigación, innovación y adopción en entornos reales.

El concepto de "Smart Cities" o ciudades inteligentes ha atraído una atención considerable en el contexto de las políticas de desarrollo urbano. Tecnologías como sistemas empotrados y redes de bandas anchas han facilitado servicios electrónicos, los cuales son cada vez más importantes para el desarrollo urbano, mientras que las ciudades asumen cada vez más un papel crítico como impulsores de la innovación en áreas como la salud, la inclusión, el medio ambiente y los negocios. 
Dichas problemáticas no son nada ajenas a la Ciudad de México, la cual tiene una población de 8.5 millones de habitantes. Donde destacan problemas de movilidad, generación de energía, contaminación, ordenamiento urbano [3]. Una de las zonas más afectadas por la problemática es Santa Fe, anteriormente esta zona era un relleno sanitario, el cual se convertiría con el pasar de los años en la zona económica - industrial más importante de la ciudad de México. El crecimiento de Santa Fe no fue planificado ni diseñado por lo que ha agravado el problema del congestionamiento vial.

Dentro de este documento se detallan cinco secciones, el capítulo dos menciona el problema que afecta la Ciudad de México, específicamente la zona de Santa Fe, cómo surgió la zona y fue desarrollándose; el capítulo tres se encuentran los retos y desafíos que tienen las ciudades inteligentes. En el capítulo cuatro, se trata de la solución implementada y por último conclusiones y comentarios que se obtuvieron en la elaboración del proyecto.

\section{Santa Fe y su problemática vial}

La ciudad de México es la capital de los Estados Unidos Mexicanos, la cual fue establecida como ciudad el 18 de noviembre de 1824 [10].

La ciudad de México ha ido creciendo a un ritmo sumamente acelerado y muchas veces sin planificación. Actualmente tiene una población que supera los 8 millones de habitantes y con una superficie que ronda los $1485 \mathrm{~km} 2$, la cual se encuentra dividida en 16 delegaciones que conforman la ciudad. Las cuales son:

1. Álvaro Obregón

2. Azcapotzalco

3. Benito Juárez

4. Coyoacán

5. Cuauhtémoc

6. Cuajimalpa

7. Gustavo A. Madero

8. Iztacalco

9. Iztapalapa

10. Magdalena Contreras

11. Miguel Hidalgo

12. Milpa Alta

13. Tláhuac

14. Tlalpan

15. Venustiano Carranza

16. Xochimilco 


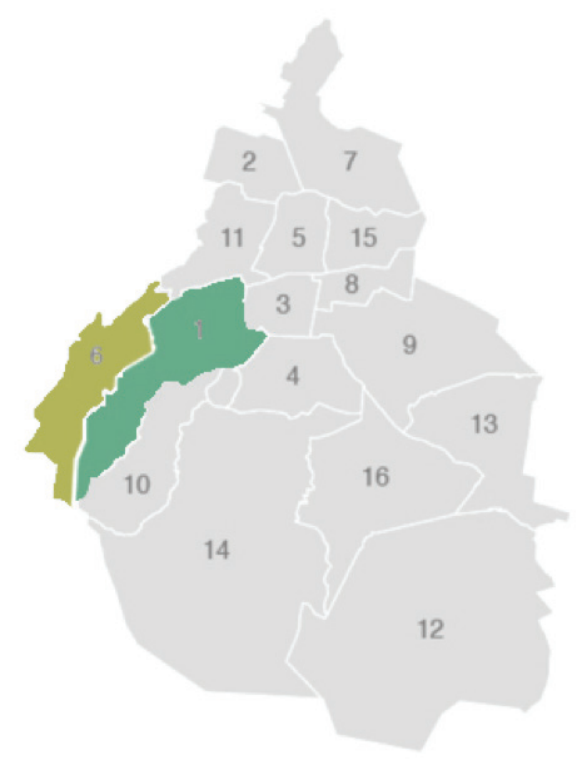

Figura 1. Delegaciones de la Ciudad de México.

La figura 1 muestra la división territorial de las delegaciones que conforman la Ciudad de México. La zona de Santa Fe se encuentra entre las delegaciones Álvaro Obregón (1) y Cuajimalpa (6):

"La zona de Santa Fe es un distrito financiero de la Ciudad de México, el cual se encuentra ubicado al lado de la Carretera Federal No. 15D México-Toluca y la autopista México-Toluca 134D." [11].

La zona de Santa Fe fue hace 30 años una zona dedicada a la extracción y/o explotación de arena; con el crecimiento que tenía la industria constructiva en la Ciudad de México provoca la sobre explotación de las minas, lo que tuvo como consecuencia la alteración de la fisiografía, estabilidad de los terrenos, la topografía y el paisaje de la zona. Posterior a eso la zona es convertida en tiraderos o rellenos sanitarios.

A finales de la década de los ochentas se propone la creación de una "Zona Especial de Desarrollo Controlado (ZEDEC)", la cual crea el Plan Maestro para el Desarrollo de Santa Fe y es cuando se aprovecha la compra de terrenos e inicia el proceso de construcciones, de forma paralela se encuentra la construcción de la Autopista México-Toluca, que de esta manera se empieza a ver el potencial y consolidación de la zona [11-13].

La zona empezó a crecer y con esto una serie de problemáticas comenzaron a surgir en la zona, principalmente por la falta de planificación y diseño a la hora de la construcción de la ciudad. Esto debido a que los desarrolladores tenían permitido un diseño individual y protagónico. Con esto surge nuevas problemáticas que afectan actualmente a la zona de Santa Fe.

En la actualidad, la zona de Santa Fe tiene el problema de insuficiencia de infraestructura para el transporte, esto se debe a la desarticulación de las áreas internas, generadas por las barreras físicas y naturales que existen en la zona. Además, se suma la ausencia de otros medios de transporte como el metro o metrobús; lo que ha provocado el fomento de utilizar el vehículo como medio de desplazamiento de personas, inclusive en distancias sumamente cortas.

La desarticulación de Santa Fe con el resto de la ciudad, y su relativa lejanía con la Zona Metropolitana del Valle de Toluca, aunado a las condiciones del terreno (desniveles pronunciados y barrancas) y a los limitados accesos/salidas existentes, ha provocado que las personas tarden 
hasta 5 veces más en sus recorridos. Se prevé que en los próximos años un incremento urbano en Santa Fe complique aún más el conflicto de movilidad ya existente.

La Encuesta Nacional de Movilidad y Transporte elaborada por el Instituto de Investigaciones Jurídicas de la Universidad Nacional Autónoma de México (UNAM) en el año 2014, revela que la mayoría de los propósitos de un viaje es ir al trabajo (46.3\%), seguido de adquirir un bien o servicio (19.1\%). La figura 2 se muestra otras causas por las cuales las personas se desplazan de un lugar a otro [1].

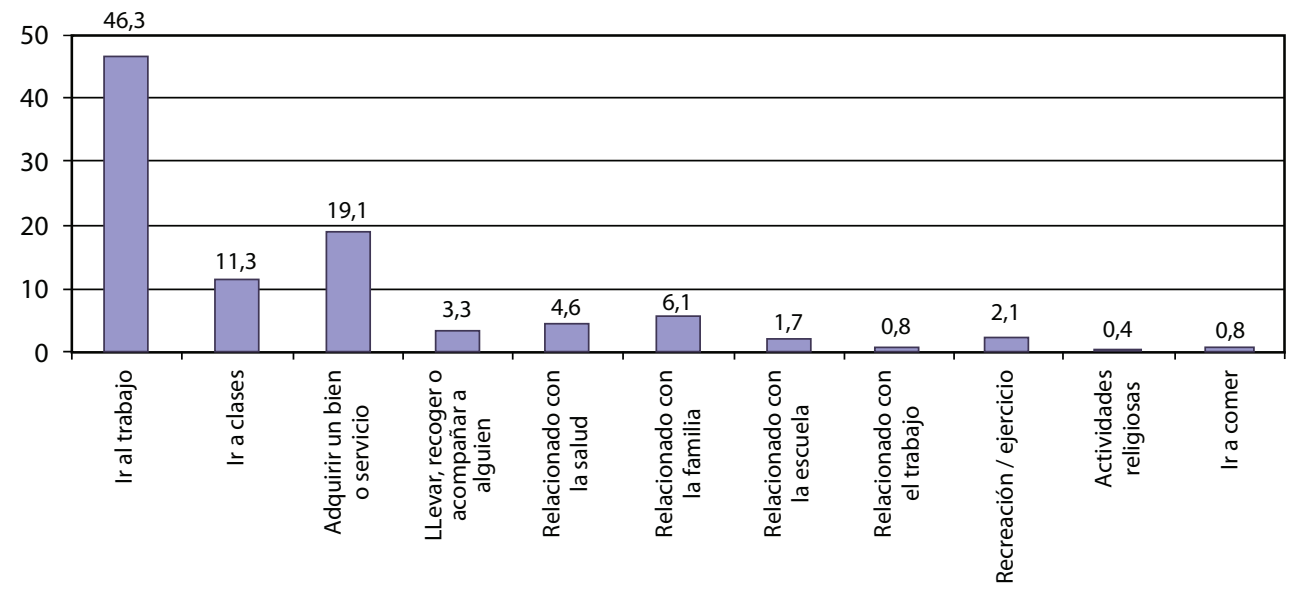

Figura 2. Propósitos de los viajes - sin considerar el regreso.

Sumado a los propósitos de viaje que tienen las personas, también se encuentra el periodo de duración de los viajes ronda los 43.6 minutos; dichas cifras también revelan que aumentan en zonas en especifico como la parte norte del país. En la figura 3 se muestra la duración de los recorridos de acuerdo con el modo de transporte utilizado [1].

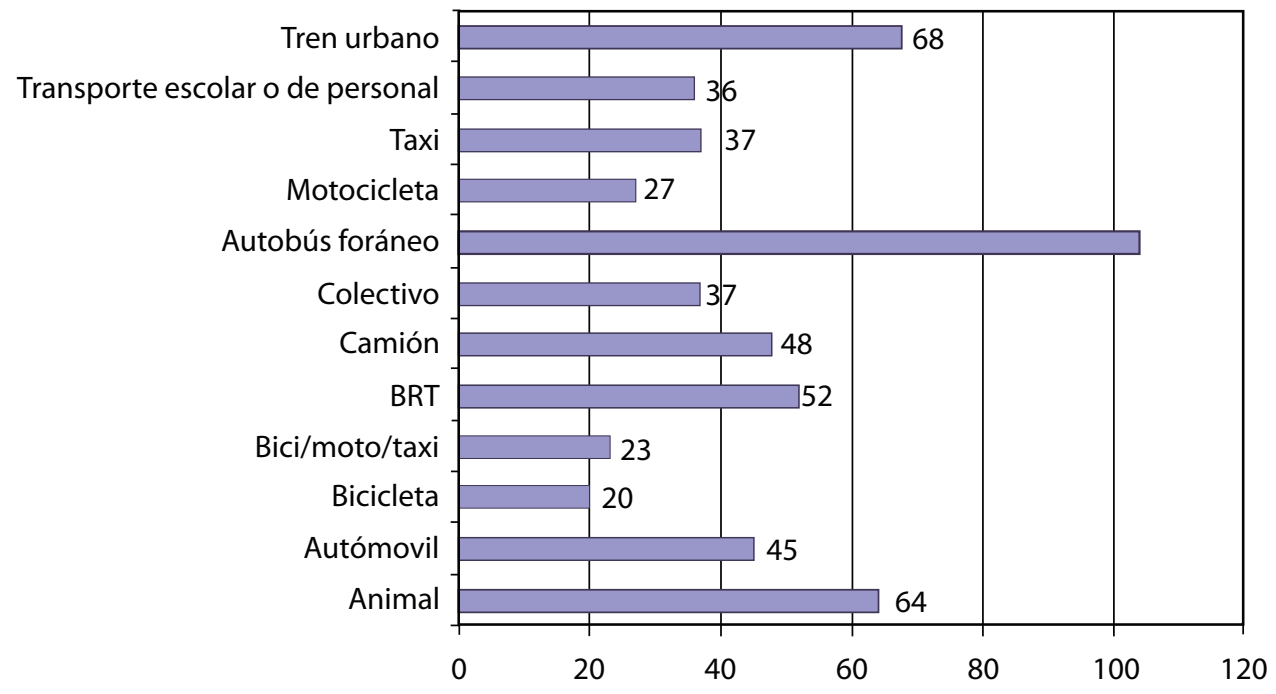

Figura 3. Tiempo de recorrido en viajes unimodales por modo de transporte. 
Además, la forma de como los usuarios prefieren desplazar de un lugar a otro. La figura 4 nos muestra un gráfico de los principales medios de transporte utilizados en la ciudad de México para desplazarse.

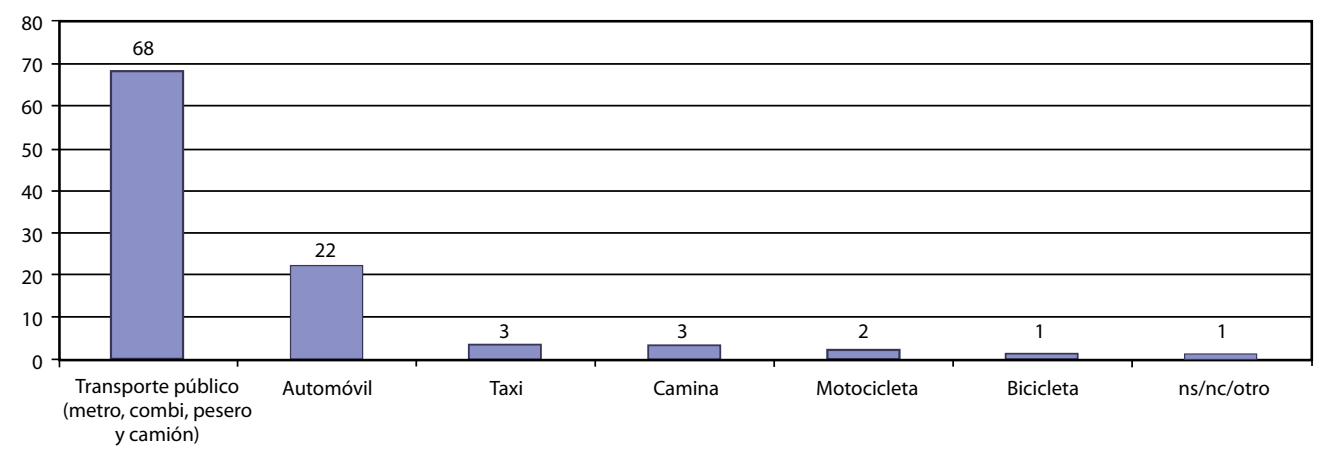

Figura 4. Principales medios de transporte utilizados para moverse en la ciudad (Porcentaje).

Con esas estadísticas proporcionadas la UNAM, es fácil identificar la problemática de la movilidad vial que existe en la Ciudad de México, además de los impactos económicos y ambientales que genera al país, como a sus habitantes. Recorridos que deberían de tardar una hora, dilatan hasta mas de una hora con treinta y tres minutos de tráfico, que conlleva a que los ciudadanos presenten otros problemas como tensión, estrés, mal humor e inclusive otras enfermedades.

\section{Desafíos y Retos Urbanos de una ciudad inteligente como México}

Existen muchos desafíos y retos para implementar tecnologías de punta y crear ciudades inteligentes. Muchos de estos desafíos tienen que ver con aspectos o intereses políticos, sociales, ambientales, económicos y tecnológicos.

\section{A. Intereses Político - Económicos}

Existen grupos elitistas que controlan o tienen miedo de perder un "statu quo" permanecen resistentes a la integración y a compartir información necesaria para crear la infraestructura básica para las ciudades inteligentes.

\section{B. Sector transporte}

El transporte es una de las áreas que se debe desarrollar, ya que presenta problemas como: diseños de espacios compartidos, donde puedan coexistir distintos usuarios/actores de transporte (peatones, ciclistas, autobuses, entre otros). Implementación de semáforos inteligentes que permiten acortar el tiempo de espera de acuerdo con el volumen de vehículos. En cuanto al transporte publico se puede ampliar (horarios y líneas) de acuerdo con las necesidades actuales de los usuarios [2].

\section{Ambientales}

Sensores que permitan el aprovechamiento al máximo de la luz solar o sensores en procesos de distribución de agua que permitan la reducción de pérdidas. Un diseño que permita una adecuada recolección de desechos y limpieza de alcantarillas. 


\section{Tecnología}

La tecnología es un gran aliado en nuestra sociedad, pero es necesario tomar medidas con respecto a seguridad y privacidad en el Internet de las Cosas (IOT, Internet of Things). En base a la seguridad es necesario aplicar políticas o diseñar sistemas que permitan asegurar que las operaciones no se vean afectadas o que la información de los usuarios no sea divulgada o difamada en ambientes distribuidos [14].

\section{Solución implementada}

Este proyecto nace con la finalidad de mejorar las rutas entre Miguel Ángel de Quevedo y Balderas hacia la zona de Santa Fe, dicha iniciativa busca que las personas disminuyan el tiempo de traslado entre sus hogares y lugares de trabajo mediante el uso de la tecnología.

El Tecnológico de Monterrey ha creado un proyecto estratégico denominado Red Nacional de Centros para la Toma de Decisiones. Esta red está compuesta por un conjunto de nodos donde cada uno de ellos existe una sala especialmente acondicionada para la toma de decisiones basada en evidencia. Esta sala es denominada Centro para la Toma de Decisiones y se caracteriza por su alta capacidad de despliegue inmersivo, donde un grupo de tomadores de decisiones pueden revisar escenarios que simulan un problema real. A través de los escenarios se logran modificar variables que representan cambios en escenarios futuros. De esta manera los tomadores de decisión pueden llegar a un consenso con base en la evidencia presentada.

El Centro para la Toma de Decisiones fue inaugurado en el Campus de la Ciudad de México en mayo 2015. Desde entonces se han realizado proyectos académicos y de investigación. En noviembre de 2016, se realizó un acuerdo de colaboración entre la Red Nacional de Centros para la Toma de Decisiones y el Laboratorio para la Ciudad de México. Este laboratorio es una entidad del gobierno de la Ciudad de México que busca mejorar los servicios ciudadanos a través de la tecnología. Uno de los objetivos de esta colaboración es el análisis de grandes volúmenes de datos para la propuesta de nuevos proyectos hacia la mejora de servicios ciudadanos en la Ciudad de México.

Es a través del Centro de Toma de Decisiones que se implementará la solución, la cual consiste en obtener los datos de los GPS que tiene cada uno de los autobuses y empezar un proceso de limpieza de datos, continuando con un análisis de los comportamientos que tienen los datos (ejemplo: cuando hay un cambio en las condiciones del tiempo, se puede presentar un patrón distinto en los datos). Una vez encontrados dichos patrones, se deberá implementar una solución o propuesta visual, la cual es analizada en el Teatro de Toma de Decisiones.

\section{A. Extracción de los datos}

El proceso de extracción de datos es realizado por el Laboratorio de la Ciudad de México, el cual facilita un archivo Excel que contiene datos como: Placa, Fecha, Día de la semana, Tiempo, Grupo de Vehículo, Nombre del Territorio, Ubicación, Geocodificación inversa, Distancia de viaje $(\mathrm{km})$, Distancia de Ubicación (Km), Mudanza tipo estatal, Dirección, Dirección de grado, Velocidad (Km/h), Latitud y Longitud.

\section{B. Limpieza y estandarización de los datos.}

De los datos suministrados por Laboratorio de la ciudad de México, son necesarios los siguientes: Placa, Fecha, Tiempo, Nombre del Territorio, Latitud y Longitud. Por lo cual el resto de datos no utilizados son descartados para no entorpecer el análisis de los mismos. 
Se debe estandarizar las fechas, esto porque el archivo Excel brindado, no contiene el formato "timestamp" y este tipo formato es necesario para ser utilizado, por lo cual se realiza una unión entre los campos de fecha y tiempo.

Se calcula la distancia a través de la fórmula de Harvesine [16], la cual consiste en sacar la diferencia entre dos coordenadas georefenciadas utilizando el radio de la tierra, para esta fórmula se considera que la circunferencia de la tierra es esférica. A continuación, se muestra la fórmula de Harvesine que se utiliza:

$$
\begin{gathered}
\mathrm{a}=\left[\sin \left(\frac{\Delta \text { latitud }_{2}}{2}\right)\right]^{2}+ \\
\mathrm{c}=2 \cdot \arctan (\sqrt{\mathrm{a}}, \sqrt{1-\mathrm{a}}) \\
\operatorname{dist}=\mathrm{R} \cdot \mathrm{c}
\end{gathered}
$$

Donde:

- dist es la diferencia de la distancia entre dos puntos.

- a es la variable que se usa para el resultado de aplicar la fórmula de Harvesine, puede utilizar cualquier otra letra.

- $\Delta$ latitud es la diferencia entre la latitud y latitud $_{1}$.

- $\Delta$ longitud es la diferencia entre la longitud ${ }_{2}$ y longitud .

- $R$ es el radio de la tierra, el cual es una constante de $6356.752 \mathrm{~km}$ aproximadamente

- c cálculo para tener el resultado en grados en vez de radianes

- latitud ${ }_{1}$ es la latitud del primer punto

- latitud 2 es la latitud del segundo punto

- longitud es la longitud del primer punto

- longitud 2 es la longitud del segundo punto

La distancia es dada en kilómetros y se utiliza además para el cálculo de las velocidades de cada uno de los registros en las placas. Para este cálculo se utiliza la siguiente fórmula:

$$
\mathrm{v}=\frac{\mathrm{d}}{\mathrm{t}}
$$

Donde:

- $\quad v$ es la velocidad

- d es la distancia, se utiliza la calculada anteriormente.

- $\quad$ t es el tiempo, el cual es calculado en cada registro, a través de una diferencia entre el registro anterior y el actual.

De esta forma se termina de realizar la limpieza y estandarización de los datos. 


\section{Visualización en un sistema multi-pantalla}

Los datos procesados anteriormente, son cargados a la herramienta "Tableau Public", esta herramienta reconoce de forma automática los tipos de datos. Una vez que los datos han sido cargados, se procede a trabajar con los datos para que a través de $n$ pantallas, se pueda comenzar a llevar a cabo un proceso de análisis a partir de un caso de estudio con datos reales.

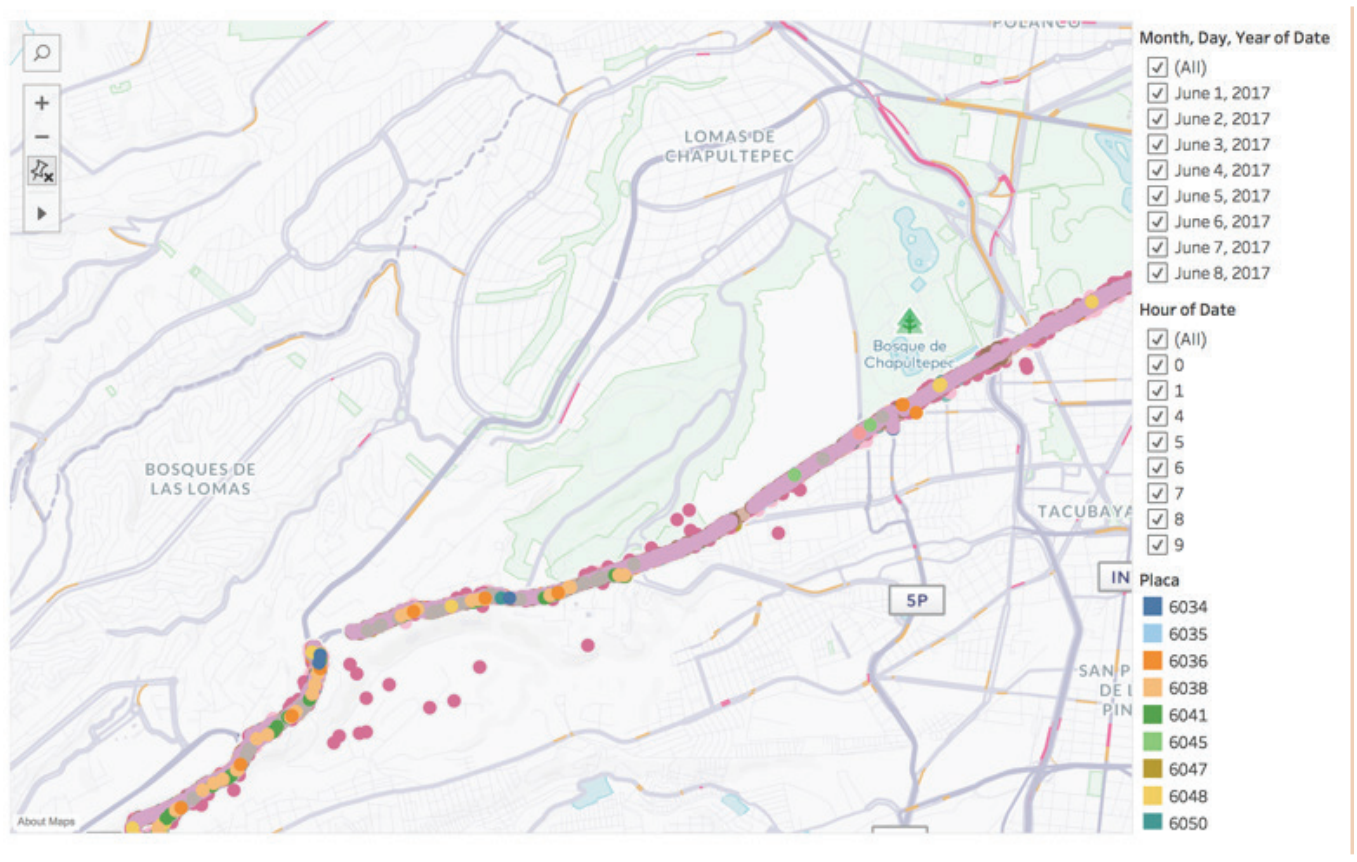

Figura 5: Recorrido de los camiones

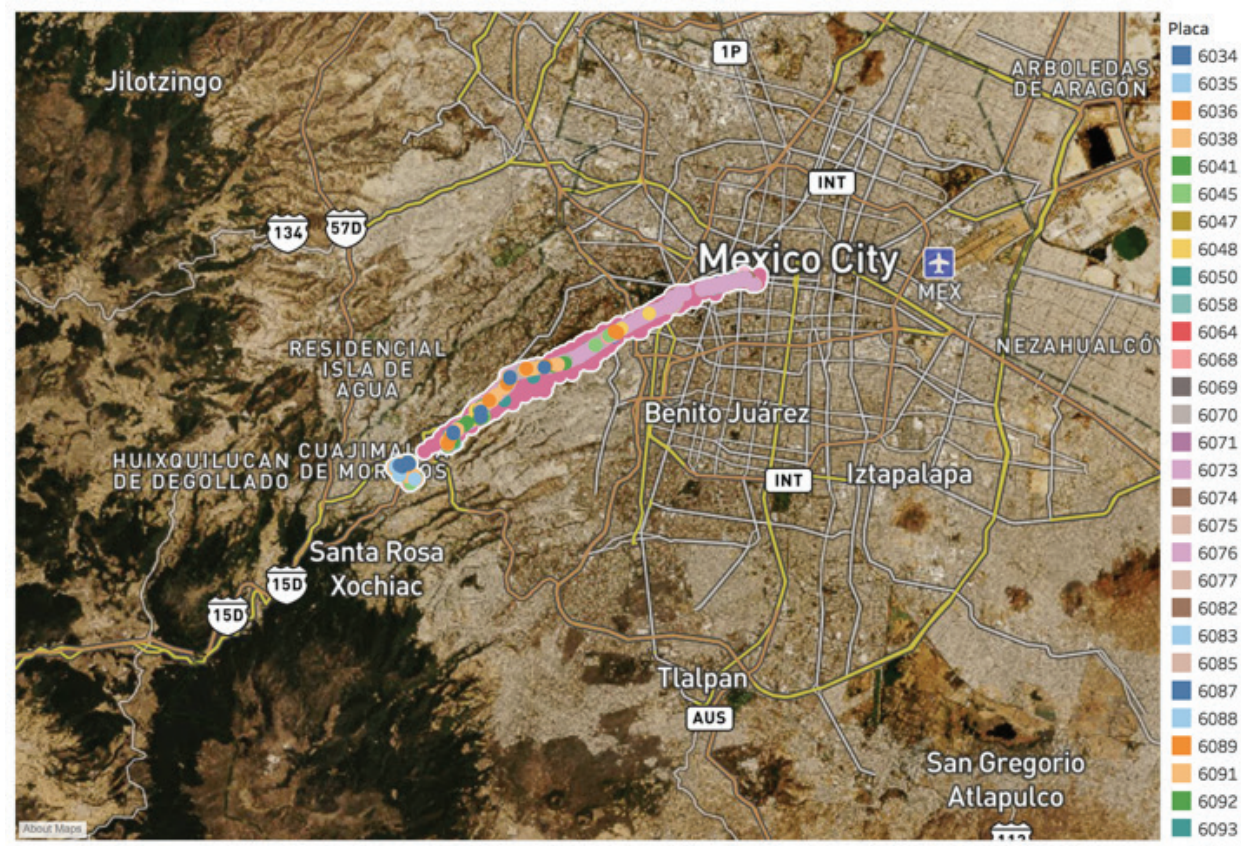

Figura 6: Recorrido Satelital de los camiones 


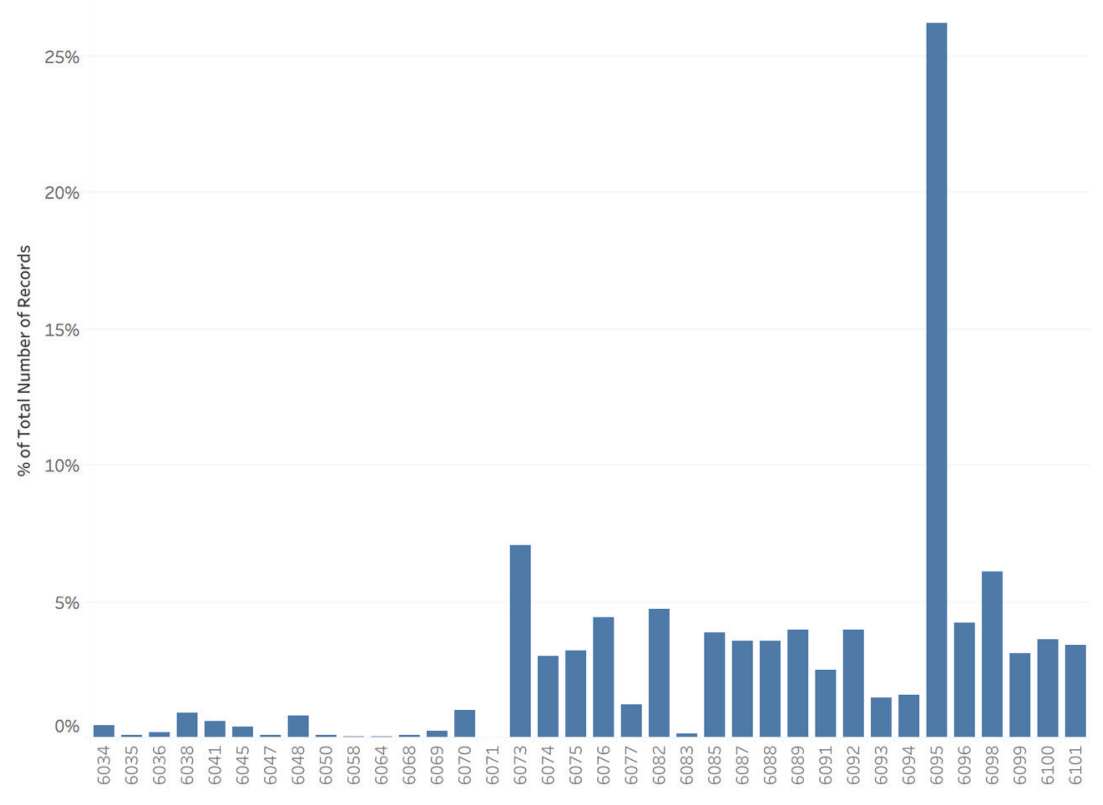

Figura 7: Placas más utilizadas

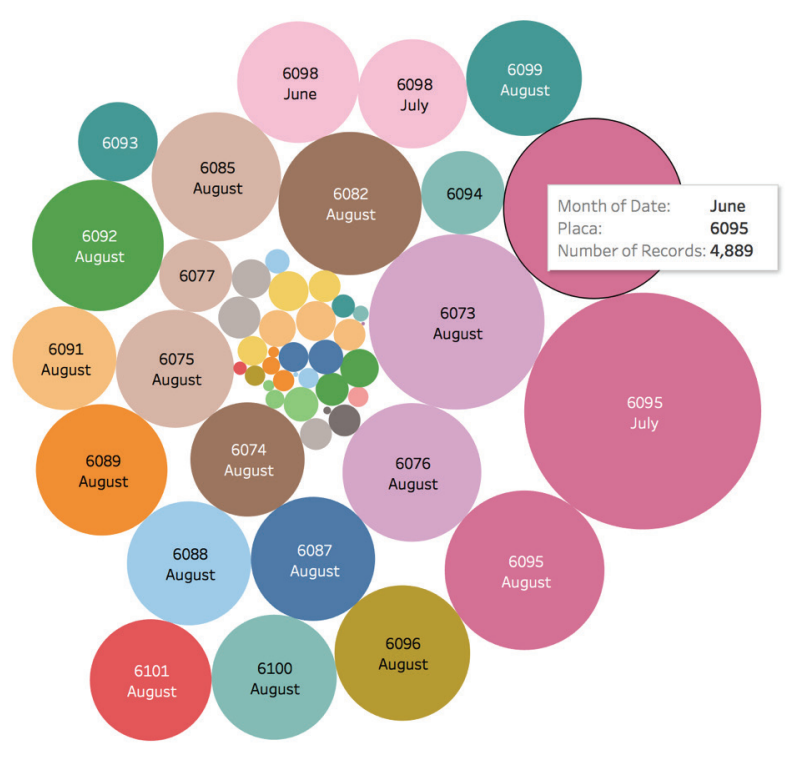

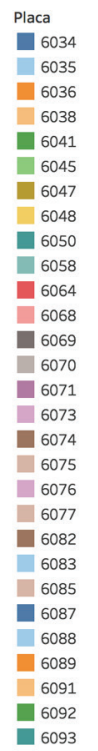

Figura 8: Placas más utilizadas por mes

La figuras de la 5 a la 8, muestran los datos que empiezan a ser trabajados de manera visual y que permite, en un sistema multi-pantalla iniciar un pensamiento crítico analizando los distintos escenarios con datos reales en un momento deseable para llegar a un acuerdo en la toma de decisiones. 


\section{Toma de Decisiones}

La Toma de Decisiones es la parte más importante, esto debido a que personas van a recibir una información tratada previamente, que le permita al usuario a través de un despliegue inmersivo de datos realizar un análisis crítico y profundo de la situación experimentada.

Una vez analizada toda la información los tomadores de decisiones empiezan a sacar sus comentarios, dudas y conclusiones para así llegar a una respuesta consensuada.

\section{Conclusiones y Comentarios}

Dentro de las conclusiones se puede mencionar que a través de grandes volúmenes de datos obtenidos de distintos dispositivos involucrados en nuestra vida cotidiana podemos analizar datos e información para la realización de procesos que optimicen y mejoren la calidad de vida de las personas. Otras conclusiones obtenidas fueron las siguientes:

- Se logra detectar que, en la Ciudad de México, conforme se acercan las fechas de pago (días 15 y 30 de cada mes), la utilización de transporte va aumentando considerablemente. Este efecto no solo incluye al transporte público, sino que también los vehículos personales/privados de los ciudadanos, lo que incrementa el congestionamiento vial.

- Otro factor que se logra detectar es que hay un mal uso o aprovechamiento de los autobuses debido a que son utilizados unos más que otros. Hubo autobuses que fueron más utilizados en un mes que otros.

- Se puede implementar un mejor control a través de una aplicación que recoja datos e información de controladores o chequeadores que se encuentren en paradas de buses que permitan ver si es necesario utilizar mayor o menor cantidad de autobuses e incluso si hay eventos en la ciudad que generen un conglomerado de personas fuera de lo normal, que saturen el servicio.

- Se logra detectar que la entrada y salida de las personas a sus respectivos trabajos o centros de estudio hace un incremento en el transporte público de forma considerable.

- Se recomienda la implementación de un sistema que permita traer los datos de manera directa de Laboratorio de la Ciudad de México, que permita una inserción de forma directa a la plataforma de Tableau Public, con la finalidad que los datos sean procesados más rápidos.

\section{Referencias}

[1] J. Aguirre Quezada. (2017). Movilidad urbana en México. Retrieved from http://bibliodigitalibd.senado. gob.mx/bitstream/handle/123456789/3391/Cuaderno\%20de\%20investigaci\%C3\%B3n\%2030\%20\%281\%29. pdf? sequence $=1$ \&isAllowed $=y$

[2] América Retail. (2018). México: Desafíos de la movilidad inteligente. Retrieved from (2018). Retrieved from https://www.america-retail.com/mexico/mexico-desafios-de-la-movilidad-inteligente/

[3] J. Arteaga. (2018). 6 problemas ambientales y sociales que colapsarán al DF • Forbes México. Retrieved from https://www.forbes.com.mx/6-problemas-ambientales-y-sociales-que-colapsaran-al-df/

[4] J. Borja. (2007). Counterpoint: Intelligent cities and innovative cities. Universitat Oberta de Catalunya (UOC) Papers: E-Journal on the Knowledge Society, 5. Available from http://www.uoc.edu/uocpapers/5/dt/eng/mitchell.pdf.

[5] S. Dirks, C. Gurdgiev, \& M. Keeling. (2010). Smarter Cities for Smarter Growth: How Cities Can Optimize Their Systems for the Talent-Based Economy. Somers, NY: IBM Global Business Services. Available from ftp://public. dhe.ibm.com/common/ssi/ecm/en/gbe03348usen/GBE03348USEN.PDF . 
[6] S. Dirks, \& M. Keeling. (2009). A Vision of Smarter Cities: How Cities Can Lead the Way into a Prosperous and Sustainable Future. Somers, NY: IBM Global Business Services. Available from ftp://public.dhe.ibm.com/ common/ssi/ecm/en/gbe0322 7usen/GBE03227USEN.PDF.

[7] S. Dirks, M. Keeling, \& J. Dencik. (2009). How Smart is Your City?: Helping Cities Measure Progress. Somers, NY: IBM Global Business Services. Available from ftp://public.dhe.ibm.com/common/ssi/ecm/en/gbe03248usen/GBE03248USEN.PDF

[8] B. Johnson. (2008). Cities, systems of innovation and economic development. Innovation: Management, Policy \& Practice, 10(2-3), 146-155.

[9] J. Marceau. (2008). Introduction: Innovation in the city and innovative cities. Innovation: Management, Policy \& Practice, 10(2-3), 136-145

[10] G. México. (2017). Gobierno. [online] Portal Ciudadano de la CDMX. Disponible en: http://www.cdmx.gob.mx/ gobierno [Accedido 28 nov. 2017].

[11] Soluciones Integrales en Tránsito y Transporte S.A de C.V (2017). Plan Maestro de Movilidad Urbana de Santa Fe. Tomo I Resumen Ejecutivo.

[12] Soluciones Integrales en Tránsito y Transporte S.A de C.V (2017). Plan Maestro de Movilidad Urbana de Santa Fe. Tomo II Informe de Campo.

[13] Soluciones Integrales en Tránsito y Transporte S.A de C.V (2017). Plan Maestro de Movilidad Urbana de Santa Fe. Tomo III Diagnóstico.

[14] C. Spera. (2016). Los desafíos de las ciudades inteligentes. Retrieved from https://www.cronista.com/columnistas/Los-desafios-de-las-ciudades-inteligentes-20160113-0004.html

[15] D. Toppeta. (2010). The Smart City Vision: How Innovation and ICT Can Build Smart, "Livable", Sustainable Cities. The Innovation Knowledge Foundation. Available from https://inta-aivn.org/images/cc/Urbanism/background\%20documents/Toppeta Report 005 2010.pdf

[16] G. Van Brummelen. (2017). Heavenly Mathematics. [online] Google Books. Disponible en: https://books.google.co.cr/books?id=0BCCz8Sx5wkC\&pg=PR7\&redir_esc=y\#v=onepage\&q\&f=false [Accedido 5 nov. 2017].

[17] D. Washburn, U. Sindhu, S. Balaouras, R. A. Dines, N. M. Hayes, \& L. E. Nelson. (2010). Helping ClOs Understand "Smart City" Initiatives: Defining the Smart City, Its Drivers, and the Role of the ClO. Cambridge, MA: Forrester Research, Inc. Available from https://s3-us-west-2.amazonaws.com/itworldcanada/archive/ Themes/Hubs/Brainstorm/forrester help cios smart city.pdf 